\title{
Pedagogical Synergy Based on the Fractal Approach: Content Selection, Training, Control (A Case Study of Mathematical Education)
}

\author{
Evgeniya Nikolaevna Gerasimova ${ }^{1}$, Sveltlana Nikolaevna Dvoryatkina ${ }^{1}$, \\ Vyacheslav Ivanovich Korotkikh ${ }^{1}$, Olga Nikolaevna Masina ${ }^{1}$, Nikolay Petrovich Puchkov ${ }^{2}$, \\ Aleksandr Vladimirovich Usachev ${ }^{1}$, Sergey Victorovich Shcherbatykh ${ }^{1 *}$ \\ ${ }^{1}$ Bunin Yelets State University, Yelets, RUSSIA \\ 2 Tambov State Technical University, Tambov, RUSSIA
}

Received 23 May 2017 - Revised 25 September 2017 - Accepted 23 October 2017

\begin{abstract}
The ideas and principles of synergistic and fractal approaches make up methodological provision for the research. An important role is also given to methods of system analysis, mathematical and visual modeling, experimental and statistical methods. The methodological basis of the article is a synthesis of synergistic and fractal approaches. The purpose of the article is theoretical underpinning and development of effective didactic mechanisms for enhancing pedagogical synergy in mathematical education by improving the key components of pedagogical process on the basis of the fractal approach. The article presents all stages of a technology for enhancing pedagogical synergy in mathematical education on the basis of the fractal approach. As a didactic mechanism of pedagogical synergy, the authors propose a set of teaching objectives and professionally oriented tasks correlated with an interdisciplinary fractal-organized base of key mathematical concepts, as well as a system for monitoring/assessment of students' knowledge on the basis of fractal methods.
\end{abstract}

Keywords: pedagogical synergy, fractal methods, structural elements of pedagogical process, mathematics training

\section{INTRODUCTION}

Mathematical education in Russia as an effective factor in the cultural, historical, and socio-economic development of society and the state, as well as an indispensable attribute of the formation and socialization of an individual over recent decades has achieved certain pedagogical results in terms of humanitarization, individualization, and informatization. Yet, this is not sufficient any more. Strategic priorities of mathematical education faced with global reforming challenges, with the pressure of globalization, with rapid development of information technology should be targeted at strengthening the applied focus and interdisciplinary integration. The conceptual idea of knowledge integrity for the purposes of personal and intellectual development, gaining a multi-discipline qualification and matching supply and demand for highly qualified personnel is of crucial importance today, as it reflects the nature of interdisciplinary knowledge, fosters a mutually enriching synthesis of various disciplines and a constructive dialogue of cultures. It is from this point of view that academic program contents, the quality of instruction, the conformity of the methodological and information support of educational process, etc. should be assessed. All these factors require a review and a reassessment of all the components of educational process in the light of new approaches and paradigms.

The leading role in this process is played by the fractal approach that has grown in the framework of an interdisciplinary synergetic paradigm and provides an integrative environment, increasing the effectiveness and quality of educational process relying on the self-organization and self-similarity of the didactic system, which includes mathematical education. Pedagogy is saturated with fractal constructions: from a multi-tier system

(c) Authors. Terms and conditions of Creative Commons Attribution 4.0 International (CC BY 4.0) apply. 


\section{Contribution of this paper to the literature}

- The research findings are novel and practically meaningful since they can serve as a basis for optimizing the structure of a syllabus, for effective organization of the learning process, as well as for identifying and assessing the synergistic effect.

- The didactic value of the proposed conceptual idea for an effective organization of mathematics learning process in higher school is that the use of fractal methods at all its stages contributes to an acquisition of complex mathematics knowledge, an activation of interdisciplinary links, a coherent perception of fractally structured knowledge constructs, and enhanced pedagogical synergy in the entire didactic system.

- This study is of a universal nature as it can become a platform for similar studies in the context of teaching other mathematics and natural science disciplines in secondary and higher schools.

'secondary school - higher school' to a complex subsystem of knowledge acquisition by educatees. Therefore, fractal principles allow one to effectively apply them to the learning process organization. According to the authors, there is a possibility to apply the fractal approach in at least three directions: to selecting, structuring the educational content, to developing learning technologies, and to assessing the knowledge acquisition by students.

In turn, distinguishing the three components of educational process determines the current need for the use of synergistic processes, the identification of effects for successful functioning and development of the entire didactic system. It should be noted that disclosing the essence of pedagogical synergy, identifying the conditions and the factors of its manifestation stimulate a teacher to more thoroughly and comprehensively examine and find the optimal opportunities for learning administration and organization.

In the general scientific sense, synergy is seen as an increased effectiveness of an activity as a result of a combination, conjunction, integration, confluence of individual parts into a single system due to the so-called systemic effect or emergence, that is, a formation of new qualities of the system obtained (Ivanov, 2016; Rayzberg, 2006). As applied to educational process, pedagogical synergy is defined by foreign authors Mascall and Rolheiser (2006) as a dynamic process of increasing the level of comprehending teaching material, strengthening the practical focus, and enhancing the coherence of an educational environment. This synergy emerges when the structural elements of a learning process complement each other rather than working as separate or conflicting objects. What is meant by synergy in mathematical education by researchers Ostashkovet al. (2016) is a 'symbiosis of the effects of an individual self-development against the background of fluctuations in objective results and stochastic nonlinear processes of self-organization of complex open systems under the influence of external parameters through coordinated effects of various factors and fundamentals in three contexts: semiotic, imitative, and social ones, applied to a system states that are far from balance'.

Distinguishing the three key components of pedagogical process - structuring and selection of mathematics educational content, the organization of teaching process, pedagogical diagnostics and control and their subsequent improvement based on the fractal approach will allow one to fully reveal the didactic potential of pedagogical synergy for effective development of an individual and society.

Comparative analysis of foreign and Russian literature that touches upon this topic, as well as everything noted above, has determined:

- the authors' objective of the research, which consists in theoretical underpinning and development of effective didactic mechanisms for enhancing pedagogical synergy in mathematical education by improving the key components of pedagogical process on the basis of the fractal approach;

- the research objectives: to study the theoretical and methodological foundations of pedagogical synergy; to develop and justify a fractal structuring methodology for a mathematics syllabus in higher school; to develop an interdisciplinary bank of teaching objectives and professionally oriented tasks through the example of the mathematical discipline 'Probability Theory and Mathematical Statistics', coordinated with fractal structuring of the syllabus and implementing the synergy of mathematics, humanities, and natural science knowledge; to develop a methodology for monitoring/assessing the mathematics knowledge of students on the basis of fractal methods; to test with experiment the effectiveness of the mathematics learning process organization in higher school based on the fractal approach.

\section{LITERATURE REVIEW AND METHODOLOGY}

In the process of the research, a synthesis of methodological approaches was used. Introduction of ideas and principles of synergistic and fractal approaches was of particular importance. Analysis of academic literature has established that application of the synergetics ideology is possible in the following areas: 
- in designing syllabi essential for the formation of worldview, methodological and interdisciplinary knowledge and skills, lateral thinking (Gapontsev et al., 2004);

- in teaching as a method of learning administration and as a method of enquiry and research of this process (Mukushev, 2008; Yushko, 2012);

- in education modernization as a methodological tool to implement integration ideas and as a pedagogical tool to establish conducive conditions for self-organization, self-development, and qualitative change of a learner's personality through the creation of an intensive information and educational environment (Knyazeva and Kurdyumov, 2011; Ostashkov et al., 2016).

The result of applying the synergetic approach to the learning process organization is an observed synergistic effect (cognitive, psychological, social), which is the effect of numerous didactic, intellectual, social, economic, and other factors involved in obtaining it. This aspect was studied in detail in the works of Krivykh (2015), Chelnokov (2010), Dvoryatkina et al. (2017), and others.

Currently, research of fractal methodology is limited to justifying the fractal structure of pedagogical objects. For example, researchers Madzhuga et al. (2015) use the fractal theory to explain the essence of such concepts as learning, instruction, self-instruction, education, self-education, development, and self-development. However, the authors Dvoryatkina and Kuznetsova (2015) see a potential practical application of the linear self-similarity concept to pedagogical constructs. This concept provides a control over the process of establishing meaningful connections of various levels and controls at the required tiers of interpenetration, preserving the invariant part of mathematics knowledge, stimulating motivation of educatees supported by dynamic activities, high visibility, and timely feedback, etc. Thus, the proposed methodological basis will create conditions for updating the synergistic effect and a formation of holistic integrating mechanisms.

\section{MATERIALS AND METHODS}

An important role in the study has been played by methods of theoretical and methodological analysis, including critical analysis of research and methodological literature on the problem under study, documents regulating the activity of higher educational institutions (educational standards and syllabi for mathematics training), studying the experience and results of pedagogical activity of key researchers. An important role has been also assigned to methods of fractal analysis, mathematical and visual modeling. Some of the conclusions and the quality of the research findings were ascertained using the experimental method. The experimental base of the research was comprised of three higher educational institutions in Russia: Yelets State University named after I.A. Bunin, Lipetsk State Pedagogical P. Semenov-Tyan-Shansky University, and Tambov State Technical University. The study was conducted during 2015-16 and 2016-17 academic years. The sample consisted of full-time students of mathematical and technical field of education aged 19 to 22 years old. The teaching experiment was carried out on check and experimental training in the mathematical discipline 'Probability Theory and Mathematical Statistics'. The experimental sample $\left(\mathrm{n}_{1}=40\right)$ was constituted by students trained with an innovative technology adoption. In the control group $\left(\mathrm{n}_{2}=41\right)$, this mathematical discipline was taught based on conventional teaching methods. Quantitative analysis of the results attained was performed using statistical methods.

\section{RESULTS}

Inasmuch as the current research is of a semi-theoretical nature, it will be more appropriate both to elaborate on the most significant scientific and theoretical results and to present experimental validation of the theoretical results.

\section{Scientific Theoretical Research Results}

The work of Smirnov et al. (2017) identifies and characterizes four stages of synergy manifestation in mathematical education on the basis of a dialogue of mathematics, information, and humanities cultures. Let us apply the approach set forth in this paper to the development of technology for updating pedagogical synergy in mathematical education by improving the key components of pedagogical process on the basis of the fractal approach. The fundamental of the fractal approach to the mathematics learning process organization is based on creating a syllabus structure closest to the natural psychological and physiological characteristics of a learner for them to acquire teaching material, and ensures its flexibility and adaptation to the unique needs of an individual.

\section{Stage I. Preparation and organization}

Primary objectives of this stage are: updating knowledge integration; designing educational thesauri of mathematical disciplines based on information-semantic and logical analysis; carrying out pedagogical assessment 
of the thesauri projects; allocation of humanities, natural-science, and professional components of the key concepts; expanding the fractal structure according to the selected vectors of a key concept development; selecting constraint parameters for growth of fractal conceptual frameworks (setting a number of iterations); revealing individual differences and preferences of students in mental process, motivation and reflection, creativity and communication activity.

The main result of this stage is structuring the educational contents using the fractal approach. The choice of a fractal set has been underpinned by the fact that it is a fractal that is a suitable construct of effective compression and packing the acquired information content in its lattice. Fractal sets are constructively richer than those traditionally used as they make it possible to receive a volume filled to the maximum degree and the greatest granularity without interpenetration. It is worth noting that with this approach, natural science knowledge is enriched with the humanities aspect, whereas humanities knowledge acquires a scientific basis for substantiating the essence by using the natural-science and mathematical apparatus and methods. Using a cyclic return to the already-learned material but at a higher qualitative level, one initiates a more intensive growth of fractal structures. Thus, the educational content is not only aimed at integration, generalization, and expansion of its scope, but also at a qualitative transformation of the personal experience of each educatee.

So, the structure of the modern curriculum for Bachelor's (Master's) program can be represented in the form of a fractal, in which educational content is scaled. One credit unit serves as a scaling unit. In terms of the fractal approach, a syllabus is scaled at the block level (basic, variable); then, at the level of modules (academic disciplines) with given parameters, their sections, subsections, etc., while building up a complex structure with multiple types of links (interdisciplinary, intra-disciplinary, inter-conceptual). Establishing boundaries in a domain and in its structure, as well as subdivision into a multitude of disciplines, techniques, methods, is analogous to the creation of fractal sets, such as Cantor set, Sierpinski triangle, Sierpinski carpet, etc.

The fractal approach can also be applied to structuring the content of key concepts that constitute the 'frame' of educational content. When developing a structure of mathematical concepts using basic properties of fractals, logical connections are established between certain concepts of a particular subject area and a wide scope of their application is provided, which makes it possible to control knowledge integration as a whole. Having established a correspondence between the conceptual volume and the fractal structure, one gets the opportunity to translate into the language of geometrical images the notion of multifacetedness and multidimensionality of exploration and theoretical comprehension of each concept. Superposition of fractals makes it possible to form an object characterized not by a certain value but by an entire range of fractal dimensions. Objective selection and adequate structuring of key concepts system can be achieved solely on the basis of a combination of academic literature and educational material analysis and an expert review. The thesauri formed in this way should become the fundamental basis for a subsequent construction of fractal hierarchical structures, including for the purpose of creating teaching packages in various disciplines.

Thus, educational-conceptual constructs are considered herein as developing self-similar structures that reflect the self-similarity of the whole in any of its divisible parts in connection with the identical scheme of constructing all the syllabi structural elements. The model of a structural element of knowledge (mathematical concept) is written as:

$$
S=\Delta \cup\left(\Delta_{1}^{1} \cap \Delta_{1}^{2} \cap \Delta_{1}^{3}\right) \cup\left\{\left(\Delta_{2}^{1} \cap \Delta_{2}^{2} \cap \Delta_{2}^{3}\right) \cup\left(\Delta_{2}^{4} \cap \Delta_{2}^{5} \cap \Delta_{2}^{6}\right) \cup\left(\Delta_{2}^{7} \cap \Delta_{2}^{8} \cap \Delta_{2}^{9}\right)\right\} \ldots
$$

where $\Delta$ is a basic mathematical concept; $\Delta_{1}^{1}, \Delta_{1}^{2}, \Delta_{1}^{3}$ are concepts formed after the first iteration (the level of secondary school mathematics knowledge) and corresponding to the subject, humanities, and natural science knowledge areas, respectively; $\Delta_{2}^{1}, \Delta_{2}^{2}, \Delta_{2}^{3}$ are concepts formed after the second iteration (the level of fundamental knowledge) and corresponding to the subject area; $\Delta_{2}^{4}, \Delta_{2}^{5}, \Delta_{2}^{6}$ are concepts formed after the second iteration and corresponding to the humanities domain; $\Delta_{2}^{7}, \Delta_{2}^{8}, \Delta_{2}^{9}$ are concepts formed after the second iteration, corresponding to natural-science field of expertise, etc. (Dvoryatkina et al., 2017)

The process of forming a structure of mathematical concepts, taking into account the allotted learning time, is considered up to the third level with a degree of the structure complexity approximately equal to 1.6. Expanding the syllabi structure by increasing the number of iterations is not worthwhile for the following reasons: a limited time allotted for the study of material; excessive complication of the basic content of an educational area.

\section{Stage 2. Content-based and technological phase}

Primary objectives of this stage are: content impletion of training modules based on objectified thesauri; structuring the bank of teaching objectives and professionally-oriented tasks, coordinated with the fractal structure of a conceptual apparatus; mastering educational-conceptual content constructs by means of mathematical and computer modeling; ensuring a high level of educational (for schoolchildren) and professional (for students) motivation; development of probabilistic thinking style and creative independence in educatees against the 
background of acquiring integrative knowledge and procedures; development of the ability to adapt and improve oneself in social communications based on a dialogue of cultures.

Forms: practical studies with the use of electronic information and educational environment, laboratory calculation classes, research projects, seminars and scientific conferences, remote discussion forums.

Tools: electronic educational complexes, data science, mathematical packages (STADIA, SPSS, STATISTICA, MAPLE, MatLab, MathCad, etc.), Web-resources.

Modus operandi: methods of active learning, independent activity, focus on the efficiency dynamics and individual training pace of educatees.

wtional material have been singled out according to a further growth of fractal sets: basic secondary school knowledge (propaedeutics); college-level fundamental background knowledge; the level of general professional training (the ability to apply knowledge in reference to the field of vocational subjects); the level of self-fulfillment in practice (personal and professional self-awareness, self-esteem, and self-development).

Let us illustrate partial filling of the bank of interdisciplinary tasks in mathematics through the example of the discipline 'Theory of Probability and Mathematical Statistics' compiled according to the fractal structure of the 'independence of events' concept (Table 1).

Advancement on the matrix makes it possible to calculate the probability of being at a certain level of assimilating the content, taking into account the depth of the fractal representation of learning elements, and to correct automatically the direction of the presented task material horizontally and vertically. Execution of most tasks at all levels both vertically and horizontally allows for filling the volume and achieving granularity of a training element to the maximum extent.

\section{Stage 3. Evaluation and correction}

This stage is characterized by:

- system integration of knowledge in setting up and solving problems in professional activity;

- performance evaluation of the presented technology by carrying out diagnostic procedures containing criteria, indicators, and tools for measuring learning outcomes and the observed synergistic effect;

- analysis of theoretical and empirical generalizations and reflexive control of the characteristics of an individual style formedness in professional activity.

Let us consider the possibility of applying fractal methods in improving the system of assessing mathematics knowledge in educatees. A symbiosis of qualitative and quantitative estimates of assimilating complex structures of learning elements is an application feature of fractal methods in pedagogical diagnostics. This approach is especially important for qualimetry of mathematical education where naturally arising multilayered abstractions of a content area do the groundwork for mastering complex knowledge constructs that set a value imperative for personal self-development.

It should be noted that the task of assessing the synergistic effect of growth in experience and personal development has not been solved previously either in conventional techniques of knowledge testing and monitoring the learning outcomes (Avanesov, 2014; Bolotov and Valdman, 2013), or in modern knowledge qualimetry systems based on technological advance (Dvoryatkina et al., 2017; Latyshev, 2009). This effect is caused by a coherent and synchronized influence of heterogeneous operation mechanisms leading to qualitative changes in a learner's personality. At the same time, a synergistic effect is manifested as an additional result in the context of scholarly knowledge integration, as well as in deepening the internal self-organization structure of mathematics knowledge.

The most universal mathematical model for assessing the synergistic effect of integrating knowledge and activity is the following model, whereby the common effect of any activity is expressed as a sum:

$$
\mathrm{E}=\mathrm{E}_{s}+\sum_{i} E_{i}
$$

where $\mathrm{E}$ is the common effect of learning and cognitive activity, $E_{s}$ is a synergistic effect, $E_{i}$ is the effect of learning and cognitive activity in assimilating the i-th element of mathematics knowledge.

Quality enrichment of mental operations through forming a hierarchy of knowledge, streamlining the content and structure of cognitive experience, strengthening communication and social interaction of actors based on a dialogue of cultures, developing research activity, an effective self-regulation of personality traits of a learner will serve as a form of expressing the synergistic effect in the context of integration of mathematics, information, naturalscience, and humanities knowledge. Yet, achieving and identifying a motivational effect is the most significant as part of a psychological effect that contributes to the effective development of the motivation sphere of an individual (Gerasimova et al., 2015). 
Table 1. Diagnostics of the quality of educational material acquisition in the discipline 'Theory of Probability and Mathematical Statistics'

\begin{tabular}{ccccc}
\hline Groups & 'There is an effect': $\mathbf{D} \geq \mathbf{1 . 4}$ & 'No effect': $\mathbf{D}<\mathbf{1 . 4}$ & 'There is an effect': $\mathbf{E}_{\mathbf{s}} \geq \mathbf{0 . 6}$ & 'No effect': $\mathbf{E}_{\mathbf{s}}<\mathbf{0 . 6}$ \\
\hline Control group & $14(34.1 \%)$ & $27(65.9 \%)$ & $16(39 \%)$ & $25(61 \%)$ \\
\hline Experimental group & $32(80 \%)$ & $8(20 \%)$ & $29(72.5 \%)$ & $11(27.5 \%)$ \\
\hline
\end{tabular}

This stage provided for individual assessment of the quality of educatees' learning and cognitive activity by two parameters - the depth of knowledge (D) and an estimate of the observed synergistic effect magnitude $\left(E_{s}\right)$. The problem of mainstreaming the qualimetric apparatus has been solved due to the fractal theory application.

The coefficient of the concept assimilation depth and its association with other concepts (the indicator of interdisciplinary linkedness of concepts) is expressed through a fractal dimension D of an information cluster flow. A fractal dimension is calculated by Hurst $\mathrm{H}$-exponent and is associated with the last simple relation $D+H=2$. Calculation of Hurst $\mathrm{H}$-exponent allows one to predict the dynamics of educational material assimilation process. The algorithm for calculating Hurst $\mathrm{H}$-exponent for a one-dimensional time series determining the number of interdisciplinary concepts associated with assimilating a key concept is described in detail by Dvoryatkina et al. (2017).

The technique for estimating the synergistic effect magnitude, based on geometric representation of the probability density distribution of a conceptual space, is also described in detail by Dvoryatkinaet al. (2017).

\section{Stage 4. Generalization and transformation}

Primary objectives of this stage are as follows:

- development of technological and content aspects in practical classes and in self-directed learning;

- expansion of the bank of teaching objectives and professionally-oriented tasks and forms of activity (project activities, laboratory calculation tasks, experimental and creative sites, research groups, etc.);

- creation of stable links and establishment of liaison between other educational institutions;

- transfer of the developed technology into the educational practice in other mathematical disciplines;

- testing the presented technology in other fields of knowledge.

\section{Experimental Findings of the Research}

The results obtained after the improvement and introduction of the technology for updating pedagogical synergy in mathematical education on the basis of fractal methods in the educational process in higher school confirm the authors' research hypothesis: improving the effectiveness of mathematics teaching and appearance of a synergistic effect herewith is possible through the improvement of the main key components of pedagogical process within the scope of the fractal approach (structuring and selection of the content of mathematical education, teaching process organization, pedagogical diagnostics and control).

As a result of the pilot study, values of the indicators characterizing the quality of material acquisition in the Theory of Probability and Mathematical Statistics among the students of the control and experimental groups have been obtained. Diagnostics of the quality of the learning and cognitive material acquisition was determined on the basis of two parameters - the coefficient of concept learning depth (D) and estimation of a synergistic effect ( $\left.E_{s}\right)$. For the purpose of statistical analysis of the empirical data, the multifunctional $\varphi^{*}$ (Fisher test) was applied, which allowed one to estimate the significance of differences between percentages of the two samples where the synergistic effect of interest was registered. Characteristic values that are thresholds for dividing the subjects into groups where 'there is an effect' and where 'there is no effect' have been established. The value of the fractal dimension $\mathrm{D}=1.4\left(\mathrm{D}_{\max }=1.5236\right)$ and the value of the synergy estimate $\mathrm{E}_{\mathrm{s}}=0.6\left(\mathrm{E}_{\mathrm{s} \max }=0.8\right)$ can be regarded as critical, and it is assumed that if the given values are above those critical in the subjects, 'there is an effect ', and if below, 'there is no effect'. Based on the empirical data processing, a complete experimental picture has been obtained (Table 2).

A statistical verification has made it possible to reject the null hypothesis, whereby the proportion of persons who exhibit the effect in question in the experimental group is not higher than in the control group $\left(\varphi^{*}{ }_{\text {emp }}=4.35>\right.$ $\varphi^{*}{ }_{\mathrm{cr}}(0,05)=1.64$ in terms of $\mathrm{D}$ and $\varphi^{*}{ }_{\mathrm{emp}}=3.1>\varphi^{*}{ }_{\mathrm{cr}}(0.05)=1.64$ in terms of $\left.\mathrm{E}_{\mathrm{s}}\right)$. Statistical verification allows for the conclusion that the improvement of the main components of pedagogical process on the basis of fractal methods proves the effectiveness of mathematics learning process organization. 


\section{DISCUSSION}

Thus, the key factor for effectively functioning mathematics learning system in the context of this study is improvement of key components of pedagogical process on the basis of the fractal approach, in particular, selecting and structuring the educational content, developing learning technologies, and a procedure for monitoring/assessing knowledge acquisition by students. According to the authors, the definitive modernization specificity of the key components of pedagogical process on the basis of the fractal approach reflected on the mathematics learning process effectiveness is as follows:

First, the proposed methodology of the mathematics syllabus fractal structuring promotes a more solid acquisition of complex mathematics knowledge, mainstreaming of interdisciplinary links with a simultaneous diagnostics of a wide range of these links within the framework of one discipline, intensification of various ways to perceive information, excluding the possibility of conceptual material verbatim learning;

Second, fractal structuring and systematization of the task material allow one to monitor and control the depth of establishing interdisciplinary, intra-disciplinary and inter-conceptual links, to meet personal educational requirements, to concentrate on the required depth of instruction material presentation and on various training programs;

Third, the introduction of fractal methods into the knowledge qualimetry system makes it possible to significantly increase the accuracy and speed of assessing the level of students' proficiency allowing for identifying a 'hidden' potential objectively existing but subjectively unknown; contributes to the identification and evaluation of a synergistic effect.

\section{CONCLUSION}

The didactic value of the proposed conceptual idea of effective mathematics learning process organization in a university is that the use of fractal methods at all its stages makes it possible to qualitatively change the teaching content, ensuring an increase in its technological level. First, the results obtained contribute to the fundamental nature of mastering a course in mathematics, which initiates the formation of a holistic view of nature, society, man, post-non-classical values, and interdisciplinary system knowledge in students. Second, obtaining guaranteed learning outcomes both in the depth of comprehending mathematics material and in terms of quantitative indicators is directly related to the harmonious development of a professional personality, their ability to quickly adjust to ever-changing environment, to do critical thinking, to see emerging challenges and to find solutions, to competently deal with information, to independently develop their intelligence.

The presented approach ensures perception coherence of fractally structured knowledge constructs with enhanced pedagogical synergy in the entire didactic system. According to the authors, the potential of the fractal approach as pertinent scientific knowledge that expands the methodological horizons of pedagogy is extremely high. In the long term, further integration of fractal and information technologies in pedagogical process is possible, which will allow for creation of a new infrastructure of information-transfer transitions of interdisciplinary interaction and knowledge synergy.

\section{REFERENCES}

Abdykarimov, B., Zhanabaev, Z., \& Mukushev, B. (2005). Synergetic concept of education for sustainable development. Alma mater, 11, 56-57. [in Russian]

Avanesov, V. S. (2014). Application of test forms in the new attestation technologies. Modern Higher School: an Innovative Aspect, 4, 4-15. [in Russian]

Bolotov, V. A., \& Valdman, I. A. (2013). Types and purpose of evaluation programs for schoolchildren's educational outcome. Pedagogy, 8, 15-26. [in Russian]

Chelnokova, T. A. (2010). Synergetic effect of pedagogical design. Man and Education, 2, 36-39. [in Russian]

Dvoryatkina, S. N., \& Kuznetsova, T. I. (2015). Application of the concept of linear self-similarity in the social and human sciences. The Psychology of Education in a Multicultural Space, 32(4), 79-87. [in Russian]

Dvoryatkina, S. N., Masina, O. N., \& Shcherbatykh, S. V. (2017). Perfection of methods of pedagogical diagnostics of mathematical knowledge control on the basis of modern achievements in science. The Psychology of Education in a Multicultural Space, 37(1), 71-77. [in Russian]

Dvoryatkina, S., Smirnov, E., \& Lopukhin, A. (2017). New Opportunities of Computer Assessment of Knowledge Based on Fractal Modeling. US-China Foreign Language, 15(7), 452-459. doi:10.17265/1539-8080/2017.07.005

Gapontsev, V. L., Gapontseva, M. G., \& Fedorov, V. A. (2004). Applying the ideology of synergetics to the formation of the content of continuous natural science education. Education and Science, 6(30), 90-102. [in Russian] 
Gerasimova, E. N., Kashko, T. V., Ostapenko, V. S., \& Savushkin, L. M. (2015) Methodical aspects of formation of educational motivation of students in the process of interactive training in high school. Bulletin of the Volgograd Academy of the Ministry of the Interior of Russia, 3(34), 122-128. [in Russian]

Ivanov, D. A. (2016). Concepts of 'synergy' and 'synergic effect': semantic and evolutionary aspects. Russian Journal of Entrepreneurship, 17(20), 2583-2594. [in Russian] doi:10.18334/rp.17.20.36917

Knyazeva, E. P., \& Kurdyumov, S. P. (2011). The foundations of synergetics: a man constructing himself and his future. Moscow: LIBROKOM. [in Russian]

Latyshev, V. L. (2009). Criteria for assessing the quality of the educational component of intelligent learning systems. Informatization of Education and Science, 3, 89-96. [in Russian]

Madzhuga, A. G., Sinitsyna, E. V., \& Filipenko, E. V. (2015). Conceptual and theoretical foundations of fractal pedagogy as a new field of social and humanitarian knowledge. Scientific Dialogue, 12(48), 450-159. [in Russian]

Mascall B., \& Rolheiser, C. (2006). Pedagogical Synergy: Linking Assessment, Curriculum, and Instruction. Brock Education Journal, 16(1), 45-61. doi:10.26522/brocked.v16i1.78

Mukushev, B. A. (2008). Synergetics in Education. Education and Science, 3(51), 105-122. [in Russian]

Ostashkov, V. N., Smirnov, E. I., \& Belonogova, E. A. (2016). Synergy of education in the study of attractors and basins of attracting nonlinear mappings. Yaroslavl Pedagogical Bulletin, 6, 146-156. [in Russian]

Raizberg, B. A., Lozovsky, L. Sh., \& Starodubtseva, E. V. (1997). Modern economic dictionary. Moscow: Infra-M. [in Russian]

Smirnov, E. I., Smirnov, N. E., \& Uvarov, A. D. (2017). Stages of technological support of the self-organization process in mathematical education of the future teacher. Yaroslavl Pedagogical Bulletin, 3, 102-111. [in Russian]

Yushko, E. N. (2012). Use of synergetic approaches for the organization of effective independent work of students. Fundamental Research, 6(1), 77-81. [in Russian]

\section{http://www.ejmste.com}

